\title{
ÉTUDE SUR LA NÉVRITE HYPERTROPHIQUE PROGRESSIVE DE DEJERINE-SOTTAS
}

\author{
O. Freitas Juniño*
}

L'étude de la Névrite Hypertrophique Progressive, bien individualisée par les observations fondamentales de Déjerine et Sottas (1893), conserve encore aujourd'hui le même intérêt clinique. Les observations publiées après les travaux classiques de Déjerine ont montré, en effet, que la maladie peut revêtir plusieurs aspects cliniques, ceux-ci soulevant des problèmes intéressants concernant surtout les parentés avec certaines affections hérédo-dégénératives du système nerveux.

Bien que le nombre des observations publiées soit relativement minime (49 cas jusqu'en 1935, selon Schaller et Newmann, dont 24 vérifiés anatomiquement), cette affection n'est pas excessivement rare; en effet, il semble que de nombreux cas passent inaperçus, confondus avec des syndromes amyotrophiques ou ataxiques d'autre nature, parce que la recherche de l'état hypertrophique des nerfs est fréquemment négligée dans les examens neurologiques habituels.

Nous rapportons ci-dessous trois cas que nous avons eu l'occasion d'observer dans le Service de M. Raymond Garcin (Division Jacquart, Salpêtriè̀re), les deux premiers se rapportant à des cas sporadiques de la maladie et le troisième présentant le caractère familial. Les deux premiers cas ont été présentés en collaboration avec MM. R. Garcin, I. Bertrand et Buge $^{19}$ à la Société Française de Neurologie, séance du 2 Mars 1950.

\section{DBSERVATIONS}

Ons. 1 - Mlle. Roy... Paulette, 32 ans, secrétaire dactylographe, vient consulter, en Novembre 1949, pour une atrophie des muscles des deux mains. A 28 ans, elle éprouva, au niveau de la main gauche, des fourmillements du bout des doigts, des crispations au niveau de la main, les doigts se contractaient en flexion sur l'objet qu'ils tenaient pendant quelques très courts instants; puis la contracture cédait, la main lâchant l'objet qu'elle tenait. Par

Trabalho realizado no Serviço do Prof. agrégé Dr. Raymond Garcin e entregue à Faculdade de Medicina dá Universidade de Paris em fevereiro de 1950, para obtenção do título de assistente-estrangeiro.

* Livre-docente e assistente da Fac. Med. da Univ. de São Paulo (Prof. A. Tolosa).

Note de lauteur - Nous remercions très vivement le Dr. R. Garcin, qui nous a si aimablement accueilli clans son Service et nous a inspiré ce travail, ainsi que le Dr. Ivan Bertrand, qui a eu l'obligeance de réaliser l'examen histopathologique des case 
temps froid, les āoigts de cette main présentaient des phénomènes de syncope locale, surtout le troisième et le quatrième. En outre, des phénomènes douloureux de type névralgique, comparés à une rage de dents, étaient ressentis à la face dorsale de la main et le bord cubital durant une demi-journée, avec quelques paroxysmes. Parfois, les mêmes douleurs siégeaient au niveau du moignon de l'épaule. Ces phénomènes ciouloureux sont beaucoup plus rares actuellement. Les mêmes phénonènes, deux ans après firent leur apparition à la main droite, en même temps qu'une légère atrophie apparut à la main gauche, qui devait aller en progressant lentement sans que la force musculaire des doigts ait été grandement compromise. Bientôt, avec le même décalage dans le temps, l'atrophie allait apparaître à la main droite sans déficit marqué de la force musculaire de la main, puisque la malade a pu continuer jusque dans ces derniers temps sa profession de secrétaire sténodactylographe. Depuis un an, la malade éprouve une fatigabilité dı membre inférieur droit avec léger steppage nécessitant le port d'une chaussure orthopédique. Quelques mois après, apparition de douleurs crampoïdes à la face postérieure des cuisses, de quelques crampes au niveau des mollets et de fournillements dans les orteils. Pas de douleurs en éclair.

L'examen des antécédents montre que la malade n'a jamais quitté la France, qu'elle a toujours vécu dans la Haute-Vienne. Il n'existe aucun cas analogue dans sa famille. Ellé est la dernière de quatre enfants. L'aîné, André, âgé de 47 ans, que nous avons pu examiner le 1-3-950, est en parfaite santé et ne présente aucun trouble pathologique, aucun signe neurologique anormal; la deuxiènıe, Rachel, est morte en 1918, à 13 ans, de scarlatine; le troisième, Victor, est mort à 20 ans, en 1930, de méningite. Les parents ou grands-parents et collatéraux n'ont jamais présenté d'affection neurologique quelconque. Les grands-parents de la malade sont issus de famille de deux enfants seulement. Pas de consanguinité. Rien de notable à noter en ce qui concerne les antécédents personnels de cette jeune fille. Un traumatisme ancien de l'index droit a laissé une déformation par arthrite traumatique de l'articulation de la phalange et de la phalangine de l'index.

A l'examen, il existe une atrophie musculaire bilatérale des mains du type Aran-1)uchenne, prédoninant à gauche (fig. 1). P'as d'atrophie au nive:u des avant-bras ni des bras. La force n'est que relativement peu atteinte en regard de l'importance de l'amyotrophie. Les fléchisseurs des doigts, les interosseux ont une force diminuée; plus marquée est la diminution de force de l'opposant du pouce, surtout à gauche. L'extension de la main sur l'avant-bras est diminuée. Les autres groupes musculaires de la luain ont une force normale. $\mathrm{Au}$ niveau des autres segments di: membre supérieur, de même qu'au niveau des muscles des épaules, la force est normale des deux côtés sauf une légère diminution de la flexion à gauche de l'avant-bras sur le bras. Les réflexes tendineux des membres supérieurs montrent une abolition des stylo-radiaux, des cubito-pronateurs, une diminution des radio-pronateurs et des tricipitaux, le tricipital droit étant plus faible à droite qu'à gauche. Il existe des troubles de la sensibilité objective au niveau des mains, des avant-bras: hypoesthésie tactile, thermique et à la piqûre au niveau des mains, surtout au niveau de la face dorsale et le long du bord cubital de la main, hypoesthésie qui va en se dégradant le long du bord cubital de l'avant-bras, plus marquée d'ailleurs à gauche qu'à droite. Il est à noter que ces troubles sensitifs objectifs existent là où les douleurs avaient siégé dès le début de l'affection. Ia sensibilité profonde est altérée: les vibrations du diapason sont émoussées aux mains et aux avant-bras. Par contre, le sens de position des doigts et des divers segments des membres supérieurs n'est pas altéré. Aux membres inférieurs, on note une émaciation à droite de la loge antéro-externe de la jambe; des pieds légèrement creux surtout à droit; une diminution de force des muscles de la loge antéro-externe de la jambe droite et des fléchisseurs des orteils. Ebauche d'orteils en marteat. Tous les autres muscles des 
membres inférieurs ont une force et un volume normaux, de même au niveau de la ceinfure pelvienne. Ia marche n'est troublée que par un léger steppage à droite. Les réflexes tendineux des membres inférieurs sont symétriquement abolis. Le réflexe cutané plantaire se fait en flexion des deux côtés. 11 existe des troubles sensitifs discrets au tact, à la piquire, au chaud, au niveau des orteils et de la face dorsale des pieds, au niveau de $I_{5}$ surtout. I es vibrations du diapason sont moins bien perçues à droite qu'à gauche au niveau des malléoles. Le réflexe pilo-moteur par malaxation du trapèze est normal des deux côtés. Par ailleurs, rien de particulier à noter. Pas de cyphoscoliose. Nerf́s crâniens normaux. Pas de signe d'Argyll. Pas de troubles de la coordination. Pas de fibrillation musculaire. Pas de troubles trophiques. Pas de troubles sphinctériens.
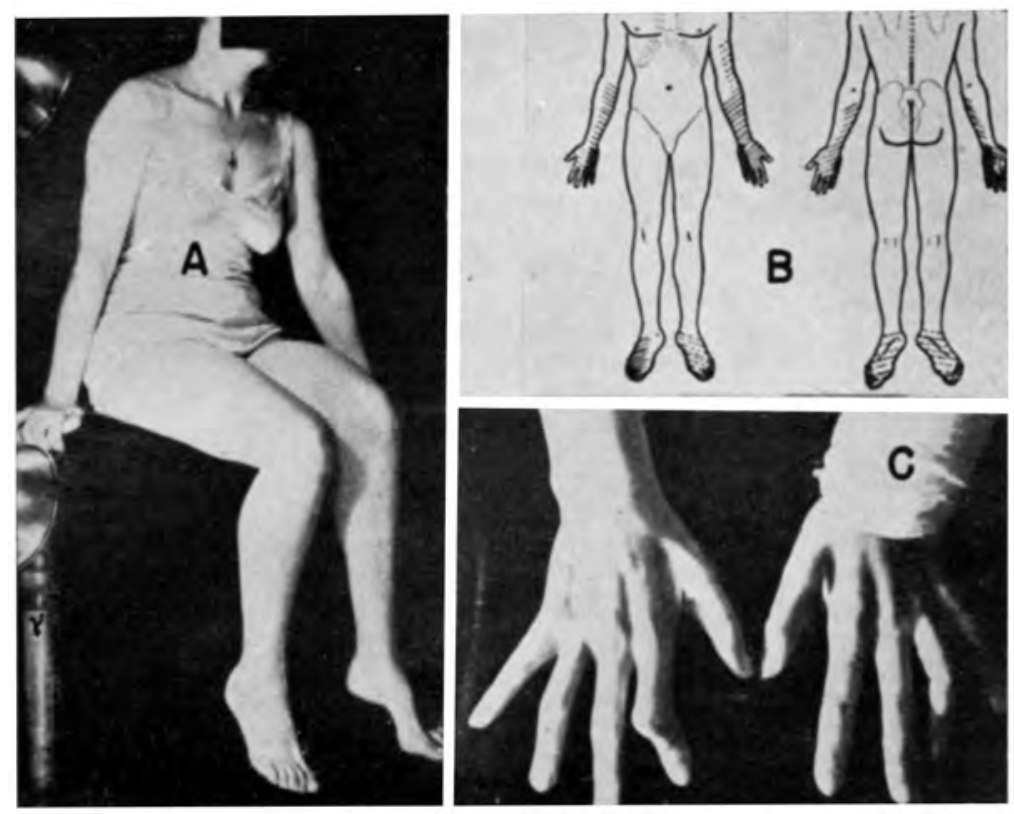

Fig. 1 -- Obs. I (Roy... P.). En A, amyotrophie des mains du type Aran-Duchenne; pieds tombants, plus à droite qu'à g:tuche. En B, hypoesthésie à tous les modes des sensibilités superficielles (en gris foncé, hypoesthésie marquée; en gris clair, hypoesthésie moderée).

L'examen des différents organes ne montre aucune anomalie. Ial tension artérielle est à 12-8, l'urcíe à $0,50 \mathrm{gr}$. Les urines ne contienent ni sucre ni albumine.

L'examen électrique (Dr. Pichaud), pratiqué le 9 Novembre 1949, montre une réaction de dégénérescence partielle accentuée, plus à gauche qu'à droite, sur tous les muscles des deux mains. Réaction de dégénérescence partielle très accentuée sur les muscles de la loge antéro-externe des jambes, plus à droite qu’à gauche et surtout sur le jambier antérieur droit. Une ponction lombaire montre un liquide céphalo-rachidien rormal: albumine, $0,25 \mathrm{gr}$. pour $1.000 ; 0,2$ lymphocytes par nillimètre cube; réaction de l'andy négative; réaction de $\mathrm{W}^{\prime}$ assermann négative; reaction du benjoin 00000.02220.00000.0. I.es réctions de Wasserman" et de Kahn dans le sang sont également négatives. 
Hypertrophie des nerfs - Hypertrophie cylindrique des nerfs cubitaux aux deux bras, du sciatique poplité externe des deux côtés, surtout à droite. Hypertrophie de la branche antérieure sensitive du radial immédiatement au-dessus de la face dorsale du poignet des deux côtés.

En résumé, il s'agit d'un cas sporadique de névrite hypertrophique progressive, la maladie ayant débuté à l'âge de 28 ans et sans antécédents familiaux. Le syndrome amyotrophyque-neurohypertrophique, accompagné de l'aréflexie tendineuse et des discrets troubles de la sensibilité superficielle, permet de poser un diagnostic formel. L'absence de signes associés (série tabétique et série cérébelleuse) classe cette observation dans le groupe des névrites hypertrophiques simples. On constate le caractère multiple et successif des amyotrophies. Elles ont frappé d'abord le membre supérieur gauche, puis le droit, et trois ans après le membre inférieur droit. Ce début par les membres supérieurs se rapproche de celui de l'observation de Long, de Dide-Courjon et de Roussy Cornil.

Ous. 2 - Mlle. Ca... Suzanne, 29 ans et demi, élève en sténodactylographie, vient consulter à la Salpétrière, le 14 Octobre 1949, pour une gêne progressive des mouvements des membres inférieurs apparue depuis l'àge de 16 ans et pour une atteinte plus récent des membres supérieurs avec amyotrophie des mains. En 1936, à l'âge de 16 ans et demi, elle éprouva de là difficulté pour courir du fait d'un début de steppage du pied gauche qui devait aller en s'accentuant lentement, mais progressivement, par la suite. En 1943, à l'âge de 23 ans, elle va consulter et l'on aurait noté dès ce moment une abolition des réflexes tendineux des quatre membres. En 1945, le même steppage apparât au niveau du pied droit. En 1948, l'apparition d'une tuberculose pulmonaire gauche la fait entrer au sanatorium où on pratique un pneumothorax. Il $y$ a un an, à 28 ans, l'araigrissement des jambes est mentionné lors de son séjour en sanatorium, indépendaminent de l'amaigrissement général. Il $y$ a un an, la malade s'aperçoit de l'atrophie du premier cspace interosseux de la main droite, sans gêne appréciable dans les mouvements du pouce. Depuis quinze jours, et c'est la raison qui motive particulièrement son examen, car elle apprend la dactỵlographie, elle s'est aperçue qu'elle ne pouvait se servir du quatriène et du cinquième doight de la main gauche pour taper à la machine.

L'étude des antécédents n'a pas permis de retrourer la moindre trace d'une affection analogue dans les ascendants ou collatéraux même éloignés. La malade est d'une famille de quatre enfants; un frère est mort de tuberculose en 1938; un autre frère de 40 ans et une sceur de 34 ans sont parfaitement bien portants. Aucun stigmate de syphilis héréditaire. Les réactions de Wassermam et de ḱahn sont négatives dans le sang. Une ponction lombaire aurait été pratiquée autre fois, qui aurait été normale, et nous n'avons pas jugé utile de la répéter. I.es urines ne contiennent ni sucre ni albumine. L'urie est à $0,35 \mathrm{gr}$.; la tension artérielle est normale.

A lexamen, trois symptômes dominent: le steppage lors de la marche, une anyotrophie diffuse mais discrète des deux membres inférieurs, plus naarquée d'ailleurs au niveau des adducteurs, enfin une amyotrophie du type Aran-Buchenne bilatérale (fig. 2). Les deux pieds sont en équin du fat d'une paralysie complète des muscles des deux loges antéro-externes. Il existe un léger déficit des muscles de la loge postérieure des jambes, plus marqué à qauche. P'aralysie des extenseurs et fléchisseurs des orteils. Il existe un certain degré de pied creux, surtout net à droite. l'as d'orteils en marteai. i noter une érythrocyanose susmalléolaire. La force des abducteurs et des adducteurs de la cuisse est diminuée; les fléchisseurs de la jambe sur la cuisse ont unc force conservée, de même que 
les extenseurs de la jambe sur la cuisse. Par contre, les psoas ont une force très diminuée. Pas de fibrillation musculaire. Hyperextensibilité au niveau de tous les segments du membre inférieur. Au membre supérieur, la force est normale tant dans l'abduction et l'adduction des bras, de même dans la flexion et l'extension de l'avant-bras sur le bras. L'extension de la main sur l'avant-hras est normale à droite, moins bonne à gauche; la flexion de la main sur l'avantbras est très dininuée dans sa force. Au niveau des doigts, les fléchisseurs ont une force normale, sauf pour les deux derniers doigts de la main gauche. Il existe de ce côté une paralysie cubitale certain, qu'atteste encore la paralysie de l'adducteur du pouce (signe de Froment). Sur les extenseurs des doigts, les extenseurs de la première phalange ont une force satisfaisante; l'extension des dernières phalanges, tout comime l'écartement et le rapprochement des doigts (interosseux), est diminué des deux côtés, particulièrement à ganche. I.es fléchisseurs, longs abducteurs, longs extenseurs du pouce, ont une force nornale des deux côtés. L'opposition du pouce aux autres doigts est diminuée. Quelques

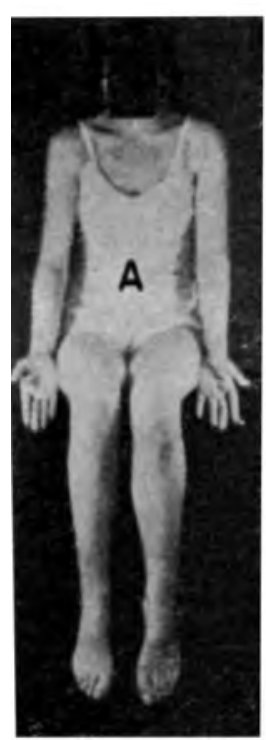

Fig. 2 - Obs. 2 diffuse et discrete des membres inferieurs, l'atrophie des mains et les pieds creux. i droite et en haut, hypoesthésie des sensibilités superficielles.

rares fibrillations musculaires apparaissent sur la face externe de l'avant-bras et le dos des mains. Il existe une atrophie des éminences des mains, modérée, surtout nette au niveau du premier espace interosseux et des muscles interosseux; une émaciation discrète de la partie inférieure de l'avant-bras, du chef postérieur du deltoïde au niveau de l'épaule; une parésie du grand dentelé du côté droit. Les réflexes tendineux des membres inférieurs et des membres supérieurs sont abolis. Le réflexe médio-pubien est aboli dans ses quatre réponses. Le réflexe naso-palpébral et le réflexe massetérin sont normaux. L'excitation cutanće plantaire ne donne aucune réponse. I es réflexes abdominaux sont normaux. Il n'existe pas de douleur au niveau des membres, pas de crampes, du moins actuclle- 
ment; la malade aurait eu quelques crampes dans les membres il y a quelques années.

L'examen de la sensibilité montre, au niveau des quatre membres, une hipoesthésie au tact, à la piqûre, au chaud, au froid, de type névritique, predominant aux extrémités et allant en s'atténuant progressivement jusqu'à niveau du coude et du genou, et cela de façon symétrique des deux côtés. Mais il s'agit de troubles discrets, révélés par l'examen systématique et jusque là ignorés de la malade. L'hypoesthésie est plus marquée au niveau de la loge antéro-externe des jambes. Le diapason est senti normalement au membre inférieur comme au membre supérieur. Mais il existe des hésitations et des erreurs dans la notion de position des gros orteils des deux côtés et peut-être aussi dans la position du pouce gauche. Les nerf́s crâniens sont normaux, les pupilles égales réagrissent normalement à la lumière et à l'accommodation. Aucun trouble de la coordination, pas de nystagmus; ajoutons enfin qu'il n'existe pas de cypho-scoliose. I.e réflexe pilomoteur par malaxation du trapèze paraît normal. l'as de troubles sphinctériens.

L'examen électrique, pratiqué à la Salpétrière (Dr. Chouraqui) en Octobre 1949 et en Janvier 1950, donne le résultat suivant: Aux membres supérieurs, les perturbations constatées ont une topographie distale symétrique; elles sont uniquement décelées dans les hypothénariens, les thénariens et le premier interosseux, qui sont le siège d'un syndrôme de dégénérescence partielle, d'importance movenne et d'intensité à peu près égale dans tous les muscles affectés. Aux membres inférieurs, on relève les séquelles de l'influence d'un processus dégénératif plus ancien à topographie également distale et symétrique. L'atteinte du territoire sciatique poplité externe a été particulièrement sévère; le nerf est inexcitable, et avec les intensités utilisées on n'observe aucune réponse à l'excitation locale des muscles de chacune des deux loges antéro-externes. Le triceps sural souffre d'une atteinte moindre, mais les réponses obtenues sont très faibles. Dans le territoire crural et dans la loge postérieure cie chaque cuisse, on ne peut retenir quine certaine diminution de l'amplitude des réponses sans lenteur galvanique.

Hypertrophie des nerfs - Le cubital est manifestement hypertrophié au bras des deux côtés, de même que les sciatiques poplités externes au niveau du col du péroné. Il existe en outre une hypertrophie manifeste de la branche sensitive antérieure du radial.

En résumé, il s'agit également d'un cas à symptomatologie essentiellement périphérique, c'est-à-dire d'une forme simple de la névrite hypertrophique progressive. Nous attirons l'attention sur: 1) L'âge du début: 18 ans; 2) L'absence d'antécédents familiaux; 3) L'amyotrophie, débutée au niveau des membres inférieurs, seulement plusieurs années après a atteint les membres supérieurs (à droite, puis à gauche); caractère multiple et successif des amyotrophies; 4) Absence des signes des séries tabétiques et cérébelleuses. En conclusion, c'est un cas sporadique, se rapprochant des cas publiés par Hoffmann, Chiarini et Nazari. Le diagnostic différentiel le plus important se pose avec l'atrophie de Charcot-Marie; mais l'état hypertrophique des nerfs fait la distinction.

Ons. 3 - Mr. Co... Casimir, 61 ans, vient consulter en Janvier 1950 pour une atrophie des muscles des jambes, troubles de la marche et des douleurs fulgurantes. Début de la maladie à 39 ans, "après intervention pour hydrocèle" (1928). Jusque là, aucun trouble fonctionnel ni organique-neurologique. Quelque temps après l'intervention, douleurs dans les mollets et apparition progressive d'une difficulté de la marche. Des douleurs fulgurantes des membres inférieurs 
ont commencé en 1930; elles sont parfois subintrantes, en particulier quand le malade a de la fièvre. En Juillet 1930, on lui fait une ponction lombaire qui est normale. L'impuissance a suivi la ponction lombaire. Vers la même époque, apparition de troubles sphinctériens, c'est-à-dire difficulté de retenir les urines. $\hat{\boldsymbol{\Lambda}}$ partir de ce moment, il constate une nouvelle aggravation de son état (marche plus difficile). En 1934, il ressent des crampes au niveau des membres supérieurs, et depuis lors nette diminution de la force musculaire de ses mains. Le froid accentue beaucoup les troubles moteurs. Ultérieurement, il constate une aggravation progressive de l'affection: troubles de la marche, surtout lors de l'occlusion des yeux, et atrophie des muscles de la jambe et des mains. Antécédents personnels: hydrocèle en 1928; blessé dans un accident d'automobile en 1938; ulcère typique du duodénum, opéré en 1947.

Examen neurologique - Amyotrophies des mains, du type Aran-Duchenne. Amyotrophie des membres inférieurs, du type Charcot-Marie (atrophie de la moitié inférieure des cuisses, atrophie des jambes, pieds tombants en varus équin, orteils en flexion). Marche en steppage bilatéral. Signe de Romberg (on note déjà une légère instabilité lorsque le malade est debout, les yeux ouverts). Force musculaire très diminuée aux extrémités des membres supérieurs, dans les territoires du cubital et du mécian (moins sur l'extension). Aux membres inférieurs,

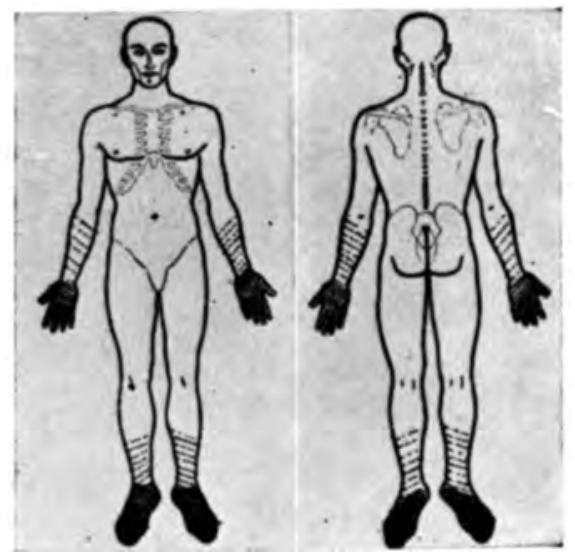

Fig. 3 - Obs. 3 (Co... C.). Troubles des sensibilités superficielles (hypoesthésie).

force musculaire abolie dans les mouvements de flexion et extension des pieds, diminuée sur la flexion et l'extension des jambes et cuisses. Légère faiblesse des fléchisseurs des jambes, surtout à gauche, à la manœuvre de Barré; léger déficit du psoas à la manuxuvre de Mingazzini. Hypotonie musculaire des membres, surtout à la partie distale; pieds ballants. Les réflexes ostéo-tendineux sont abolis aux membres inférieurs et aux membres supérieurs, sauf le tricipital. Réflexe cutané plantaire aboli. Réflexes crémastériens superficiels présent à gauche et réponse perturbée, à droite, par une intervention sur hydrocèle; réflexe crémastérien profond un peu faible à gauche; les réflexes cutanés abdominaux supérieurs sont très nets. Sensibilité subjective: douleurs fulgurantes dans les membres inférieurs; dans les membres supérieurs, douleurs plus continués, plus pénétrantes et plus nettement localisées (surtout dans le bras). Sensibilité (fig. 3): hypoesthésie tactile dans les mains et aux pieds; hypoesthésie moins importante aux jambes et aux avant-bras. Hypoesthésie discrète, à la sensibilité à la piqûre, dans les mains, pieds, jambes et avant-bras. Hypoesthésie thermique plus accentuée aux mains, aux pieds, et moins accentuée sur les jambes et avantbras. Notion des attitudes segmentaires conservée. Les vibrations du diapason 
sont diminuées sur les crêtes iliaques, les rotules, et ne sont pas perçues aux chevilles et aux orteils. Il n'y a pas d'astéréognosie. Pas de troubles de la coordination, pas d'adiadococinésie; pas de nystagmus. Les extrémités sont ïroides et cyanosées; hypohydrose aıx extrémités inférieurs. Petit retard à la miction; impuissance depuis 20 ans. Paires crâniennes normales, sauf hypoacousie de l'oreille droite, remontant à longtemps. Légère inégalité pupillaire $(O D<O G)$; irrégularité du contour des pupilles; à la chambre noire, comme à l'éclairige du jour, les pupilles gardent un diamètre moyen de $5 \mathrm{~mm}$. environ (pas de myosis); abolition totale du réflexe photomotcur des deux côtés; réactions pupillaires à la convergence-accommodation très affaiblies; il existe donc une dissociation pupillaire du type Argyll-Robertson, avec rigidité pupillaire, irrégularité pupiliaire, sans myosis. Fundi oculi normaux. $\mathrm{VOD}$ et $\mathrm{VOG}, 9 / 10$.

Hypertrophie des nerfs - On a trouvé alu nerf cubital (dans son tronc), au sciatique poplité externe, et quelques rameaux sous-cutanés, au nerf musculo-cutané (rameaux cutanés antibrachial), au nerf fémoro-cutané et rameaux du péronier superficiel.

Examen électrique (Dr. P. Mathieu) - Réponses qualitatives normales au niveau de la ceinture scapulaire, des muscles des bras et avant-bras. Diminution considérable de l'amplitude des contractions de l'ensemble des muscles des mains, qu'il s'agisse de courant continu ou de courants bref's, ou d'excitations par le nerf; cette diminution est particulièrement marquée pour les muscles thénariens (fibres de l'opposition du pouce). Fn outre, on note la présence certaine de quelques fibres lentes, en particulier au niveau des premiers espaces interosseux, des muscles hypothénariens; il s'agit donc d'atteintes périphériques partielles accentuées, avec destructions importantes non entièrement stabilisées, donc encore évolutives dans une certaine mesure. Au niveau des membres inférieurs, par contre, on ne trouve pas de fibres lentes, l'état paraît stable, les destructions sont sûrenıent très importantes. Elles intéressent surtout les territoires sciatique po-

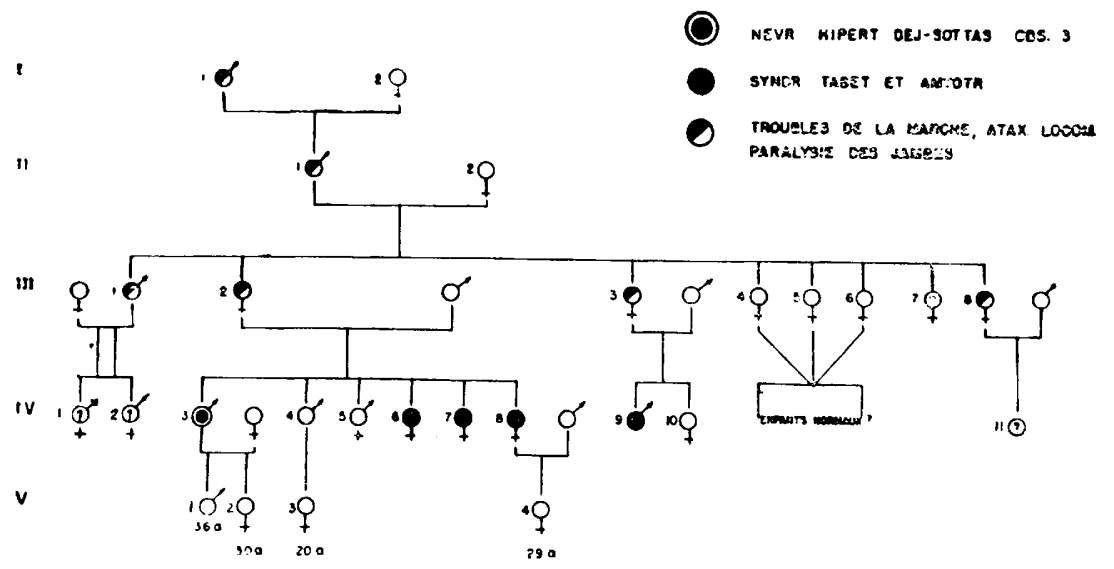

Fig. 4 - Arbre généalogique de la famille Co...

plité externe: ici, il n’y a de réponse directe et par le nerf, et de faible amplitude, qu'au niveau des péroniers. Dans les territoires sciatique poplité interne, on n'observe une absence complète des réponses qu'au niveau des muscles plantaires; les réponses des jumeaux et soléaires sont assez amples. Enfin, diminution de l'amplitude des contractions des fibres des $1 / 3$ inférieurs des quadriceps. 
Examen hématologique -. Globules rouges 3.400 .000 par $\mathrm{mm}^{3}$; globules blancs 7.600 par $\mathrm{mm}^{3}(63 \%$ de polynucléaires neutrophiles, $1 \%$ d'éosinophiles, $10 \%$ de lymphocytes, $24 \%$ de cellules mononucléées de taille moyenne et $2 \%$ de monocytes). Réaction de Bordet-Wassermamn négative.

Les renseignements sur la famille Co... montrent des cas analogues (fig. 4):

Génération $I$ - Cas I-1, décédé en 1892, à l'âge de 81 ans. Marchait à l'aide de deux bâtons. On ne peut préciser la nature des troubles de la marche.

Génération $I I$ - Cas II-1, décédé vers 1898, à l'âge de 84 ans environ, dess suites d'une maladie infectieuse ( $\vdots)$; marchait depuis de nombreuses années ave: difficulté, à l'aide de bâtons; il était complètement "instable" et présentait un steppage important; il eut 8 enfants, dont 4 certainement malades (cas III-1, III-2, III-3 et III-8); un, mort à 8 ans (III- 7 ).

Génération $I J I$ - Cas III-1, décédé en 1946 à l'âge de 78 ans; marié, était père de 2 fils décédés entre 30 et 40 ans (cas IV-1 et IV-2); a été atteint d'ataxie locomotrice vers la t.0ème année; il marchait à l'aide de 2 bâtons dians les dernières années de sa vie. Cas III-2, décédé en 1922, à l'âge de 52 ans, d'une broncho-pneumonic; a été atteint des membres inférieurs vers l'âge de 35 ans et à 42 ans avait les jambes presque totalement paralysées; a eu six enfants, dont quatre certainement malades (cas IV-3, IV-6, IV-7, IV-8). Cas III-3, âgée denviron 77 ans; membre; inférieurs paralysés depuis de très longues annćes; mère de 2 enfants (cas $I V-9$ et IV-10) dont l'aîné ( IV-9), âgé de 50 ans environ, est également gravement atteint d'ataxie. Cas III-4, III-5 et III-6, respectivement âgés de 73 ans, 69 ans et 66 ans; paraissent sains. Cas III-7, décédé à l'âge de 9 ans environ, vers 1887 , était devenu infirme à la suite d'une chîte, paraît-il. Cas III-8, atteinte de graves troubles de la marche vers la quarantaine; un fils (cas IV-11) sans aucun renseignement.

Génération IV - Cas IV-3, c'est le cas étudié dans l'observation; deux enfants de 36 et 30 ans qui ne presentent aucun trouble neurologique jusqu’à ce jour, aux dires du malade. Cas IV-4, ne semble pas présenter de troubles de la marche, mais il devait avoir les pieds plats. Cas IV-5, a fait la guerre 1914-18; pieds plats; décédé à 54 ans à la suite d'opération (perforation gastrique). Cas IV-6, 56 ans; début de l'affection en 1930 par des douleurs fulgurantes aux membres inférieurs; en 1948, les douleurs ont diminué, mais les troubles de l'équilibre et de la marche sont assez marqués; mictions impérieuses et incontinence rocturne; amyotrophie prononcée des deux membres inférieurs, atrophie des mains, présence de signe d'Argyll-Robertson et hypertrophie du sciatique poplité externe (examen pratiqué par le médicin traitant). Cas IV-7, 55 ans; douleurs fulgurantes intenses surtout atu niveau des membres inférieurs; signes de la série talbétique avec en plus amyotrophie très marquée des deux membres inférieurs; pas de troubles sphinctériens; porte un corset depuis l'âge de 30 ans, ayant été plâtrée pour mal de Pott (?). Cas IV-8, 52 ans; début de l'affection à l'âgr de 32 ans; douleurs fulgurantes des deux menbres inféricurs et sižnes de la série tabétique; troubles marqués de l'équilibre; anyotrophie des jambes avec steppage; accuse parfois un peu d'incontinence urinaire.

En résumé, nous voyons une famille où plusieurs générations sont atteintes, l'affection présentant un caractère dominant. Le cas étudié (IV-3) et une sœur du malade (IV-6), et probablement les autres membres signalés, présentent essentiellement un tableau amyotrophique associé à des signe: de la série tabétique. Dans les cas IV-3 et IV-6, l'hypertrophie des nerfs a été bien mise en évidence. En présence d'une telle symptomatologie, le diagnostic de névrite hypertrophique progressive type Déjerine-Sottas s'impose. Il est cependant intéressant de noter l'âge de début de la maladie 
(aux environs de la quarantaine), ce qui l'oppose aux cas classiques de Déjerine, qui ont commencé dans l'enfance. Le seul diagnostic différentiel à soulever est celui de tabès amyotrophique; les cas publiés par Gombault et Mallet, Mme. A. van Leeuwen et Moreau sont du même type. Les antécédents familiaux, la distribution topographique des amyotrophies, la négativité des examens du sang et du liquide céphalo-rachidien et surtout l'hypertrophie des nerfs sont des arguments suffisants pour l'établissement du diagnostic exact.

\section{EXAMEN HISTOLOGIQUE}

Les études de Déjerine, P. Marie, Bertrand, Souques ont permis de bien préciser le tableau anatomo-pathologique de la névrite hypertrophique: à côté des lésions interstitielles' (hyperplasie du tissu conjonctif), les altérations essentielles de la gaine de Schwann (schwannite).

Dans les deux premiers cas que nous publions une biopsie de la branche sensitive du radial a été pratiquée (Prof. Leger) et M. Ivan Bertrand nous a fourni les résultats de l'examen histologique qui a mis en evidénce les lésions caractéristiçues du processus:

Obs. 1 (fig. 5) - "Le périnèvre, manifestement très épaissi, est constitué par un épais feutrage de fibres collagènes et par de rares éléments fibroblastiques. I'endonèvre montre peu d'anomalies; sur coupes transversales cependant, il présente une densité anormale et enserre étroitement les gaines nerveuses. La lésion essentielle est constituée par un épaississement des gaines de Schwann, qui montrent une structure feuilletée, généralement centrée par un cylindraxe. Sur coupes transversales, cette disposition se traduit par des aspects en bulbe d'oignon. Il est fréquent de voir plusieurs neurites englobées par une même formation lobulée. Ia coloration de Loyez révèle une réduction numérique considérable des gaines myéliniques. On peut évaluer grossièrement à $1 / 10$ la proportion des gaines subsistantes. Encore celles-ci sont-elles très altérées: calibre variable, état moniliforme, atrophie, fonte granuleuse, dégénérescence bulleuse, etc. L'imprégnation de Gros-Bielchowsky confirme l'étendue des lésions neuritiques; on peut dire que les altérations cylindraxiles paraissent étroitement solidaires des dégénérescences myéliniques. I a méthode de Gros révèle en outre l'existence d'un certain nombre de neurites myéliniques circulant dans les gaines de Schwann hypertrophiées".

Obs. 2 (fig. 6) -- "Les constatations anatomiques sont comparables à celles du cas précédent. Des conditions techniques un peu différentes nous ont cependant permis quelques constatations nouvelles. Sur coupe transversale, le prélèvement se montre constitué par 4 fascicules nerveux. Seul le plus volumineux montre des images nettes de schwannite hypertrophique, en ratison de l'épaississement marqué de l'endonèvre, qui dissocie les formations schwannique slobulées. Cet aspect manque entièrement sur les trois autres fascicules; bien que l'hypertrophie schwannique soit indéniable, le tassement des gaines rend l'interprétation plus déīicate. Signalons également l'absence complète d'hypertrophie au niveau du périnèvre. Par contre, le tissu conjonctif assez lâche qui unit les divers faisceaux nerveux est considérablement épaissi. I es dégénérescences myéliniques et axonales varient considérablement en intensité d'un faisceau à l'autre; les altérations les plus importantes correspondent aux aspects caractéristiques de schwannite hypertrophique". 
"En conclusion, le diagnostic de névrite hypertrophique s'impose dans les deux cas, en raison des lésions si spéciales de schwannite, de l'hypertrophie de l'endonèvre ou du périnèvre et des dégénérescences axo-myéliniques lentement évolutives".
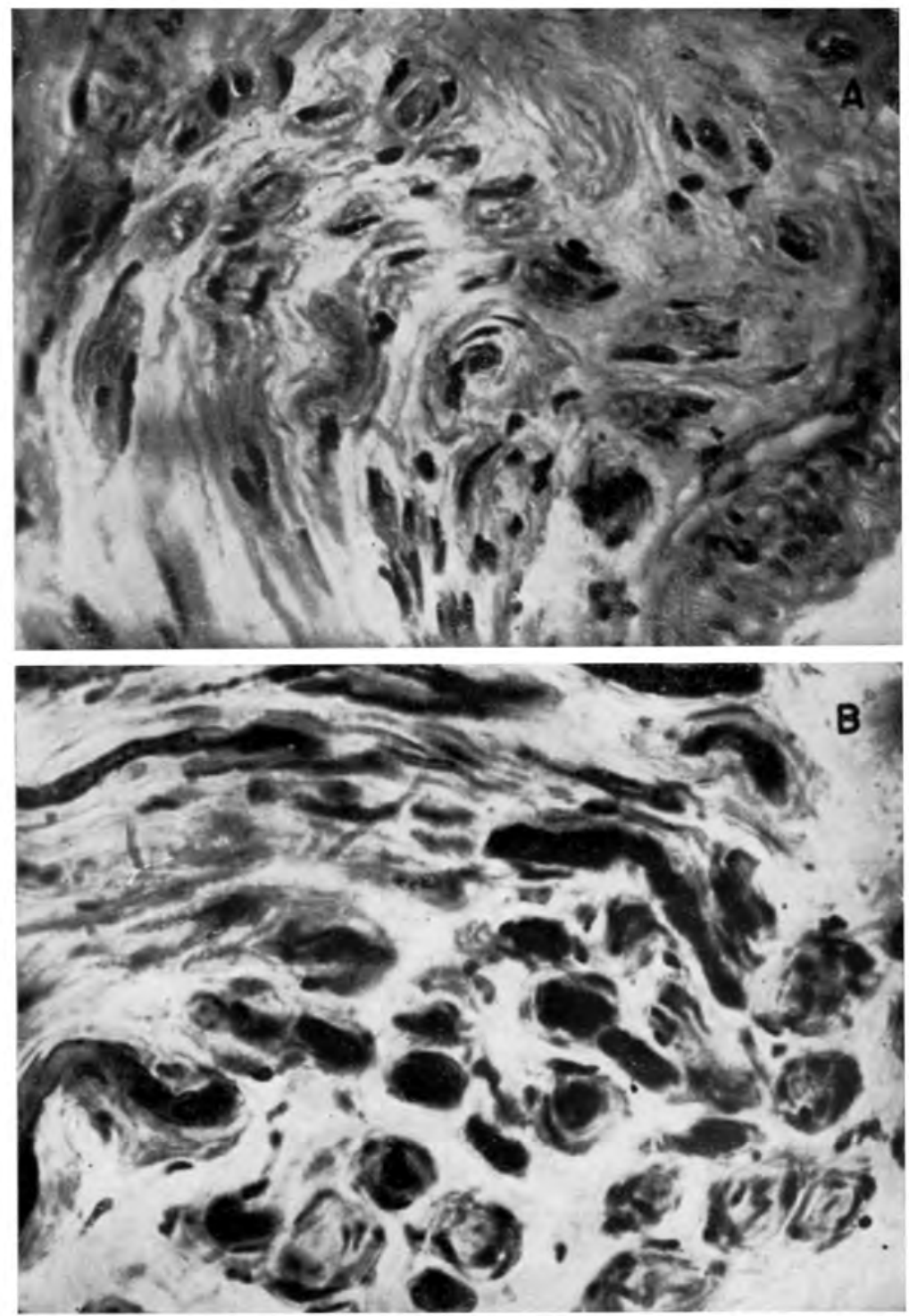

Fig. 5 - Obs. 1 (Roy... P.). Examen histologique du nerf radial: en haut, coloration par l'hemateine-eosine; en bas, coloration de Loyez. 


\section{RAPPEL HISTORIQUE}

Des observations remontant à une période allant de 1855 à 1893 et publiées comme "Atrophie musculaire progressive", "Amyotrophie Aran-Du-
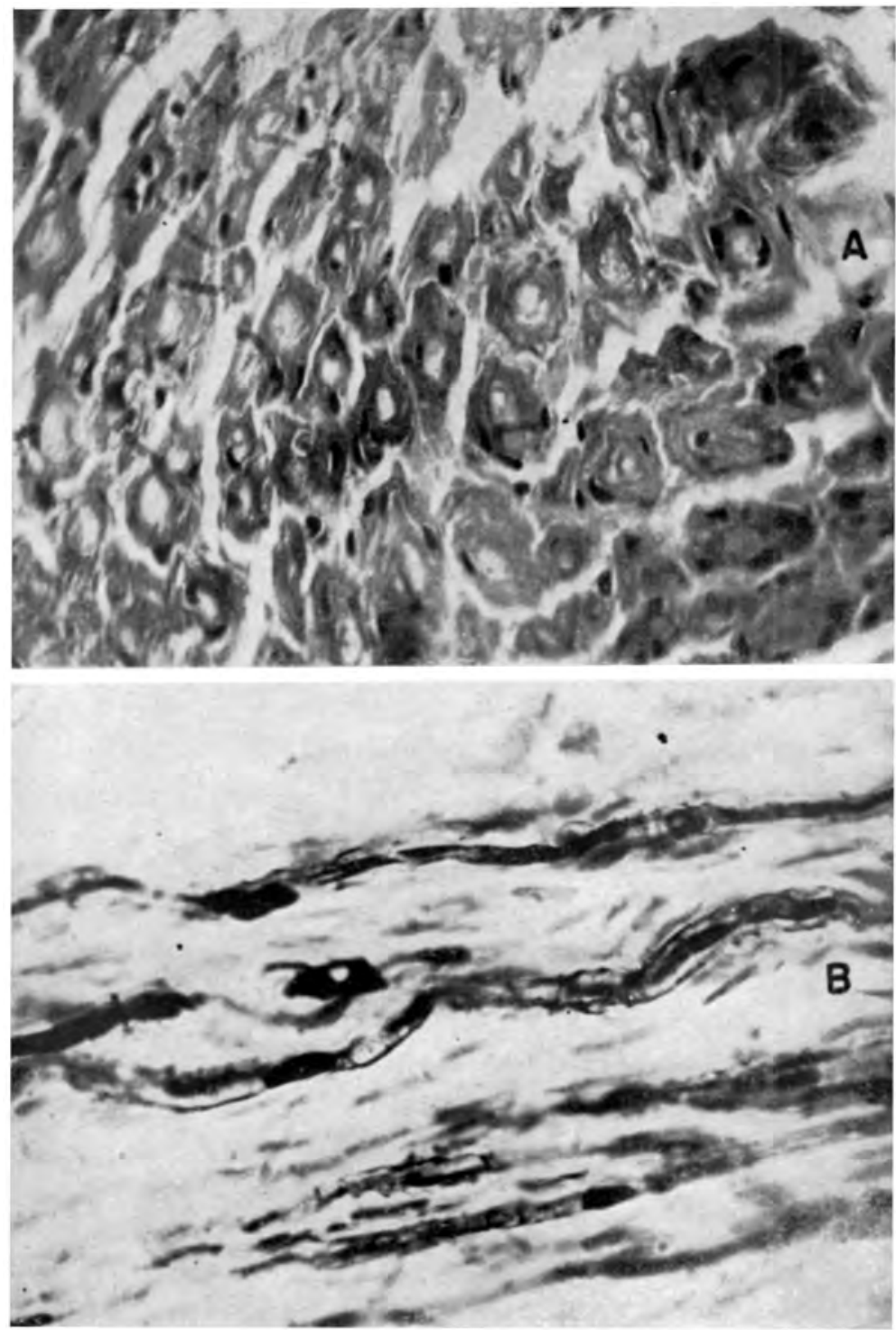

Fig. 6 - Obs. 2 (Ca... S.). Examen histologique du nerf radial: en haut, coloration par l'hemateine-eosine; en bas, coloration GrosBielchowsky. 
chenne", ou "Tabès amyotrophique", avaient révélé, à l'examen anatomopathologique, la présence d'un état hypertrophique des nerfs périphériques.

Ce tableau, néanmoins, ne fut pas considéré par ces auteurs comme l'expression d'une entité morhide autonome. Ainsi, dans un cas d'atrophic musculaire progressive observé par Virchow (1855), ayant un caractèrc héréditaire et débutant à 21 ans, les nerfs périphériques "non seulemen" n'étaient pas atrophiques, mais quelques-uns même étaient épaissis, bleuâtres; sur les coupes, on voyait une raréfaction des fibres nerveuses qui parfois atteignaient une épaisseur anormale au milieu d'une gangue conjonctive riche en noyaux, surtout dans les espaces dénudés de fibres".

Friedreich. en 1873, dans deux cas d'atrophie musculaire progressive qu'il a étudiés, a constaté: dans la première observation, atrophie localisée dans les muscles des jambes et de la main droite, une "atrophie des fibres nerveuses et une infiltration interstitielle par du tissu conjonctif riche en noyaux: les gaines de Schwann sont notablement épaissies". Dans la deuxième observation (amyotrophie progressive ayant débuié dans l'enfance et ayant un caractère familial), les troncs nerveux périphériques présentaient "un névrilème épais et un tissu conjonctif abondant, riche en noyaux, autour de fibres nerveuses plus ou moins altérécs".

En 1889, Gombault et Mallet ont publié l'observation d'un malade de 58 ans dont les troubles avaient commencé à 7 ans et qui présentait le tableau d'une ataxie associée à des atrophies musculaires, une aréflexie tendineuse, graves altérations de la sensibilité aux quatre membres, déformation de la colonne vertébrale, etc. À l'autopsie, il existait, à côté de la sclérose des cordons postérieurs, une sclérose hypertrophique des racines et des "nerfs périphériques volumineux avec sclérose conjonctive annulaire, engaînant les fibres".

En 1891, Nonne a décrit, sous le nom de "poliomyélite antérieure chronique", un cas où l'histologie retrouvait, "dans les nerfs périphériques, mélange de fibres normales, de fibres dégénérées et de fibres entourées de couches concentriques comme les écailles d'un oignon". Comme l'a signalé Long, le dessin qui accompagnait le travail de Nonne révèle sans aucun doute la disposition caractéristique des gaines de Schwann que l'on rencontre dans la névrite interstitielle hypertrophique.

Déjerine et Sottas, en 1893, eurent les premiers le grand mérite de considérer comme entité morbide autonome cette atrophie musculaire accompagnée d'hypertrophie des nerfs, confondue jusqu'alors avec des processus amyotrophiques et ataxiques d'autre nature. Ils l'ont dénommée "névrite interstitielle hypertrophique et progressive de l'enfance", et en décrivant leurs caractères, ils ont indiqué que l'affection relevait "d'une lésion parîiculière des nerfs périphériques, névrite interstitielle hypertrophique ascendante et se prolongeant dans les cordons postérieurs". "Cette affection se présente en clinique". écrit Déjerine, "avec les caractères suivants: début dans le bas âge ou la seconde enfance, par de l'atrophie musculaire des extrémités avec altérations de la sensibilité et douleurs fulgu- 
rantes, cypho-scoliose, hypertrophie et dureté des troncs nerveux, absence de troubles trophiques cutanés. Puis apparition de symptômes d'incoordination motrice avec signe de Romberg, myosis avec réaction lumineuse lente ou abolie, signe d'Argyll-Robertson et nystagmus dynamique. Le tableau clinique présenté par les malades est alors celui d'un atrophique doublé d'un ataxique. Cette affection est en général familiale et doit être considérée comme une maladie d'évolution relevant d'une maladie de développement du système nerveux périphérique, opinion que vient encore corroborer l'état de dégénérescence mentale présentée par nos malades".

Dans une publication ultérieure, et en réponse à des critiques faites sur l'isolement nosographique de la névrite hypertrophique (Marinesco, Raymond, Beduschi, ctc.), Déjerine a insisté sur l'importance primordiale de l'hyperirophie des nerfs périphériques pour l'établissement du diagnostic, caractère suffisant pour affirmer l'autonomie de la maladie.

En 1906, une forme spéciale de névrite hypertrophique progressive est décrite par Pierre Marie, qui présent à la Société de Neurologie deux frères d'une famille de sept enfants tous frappés de la même affection. Ils avaient plusieurs des symptômes de la maladie de Déjerine-Sottas (atrophie musculaire, aréflexie tendineuse, cypho-scoliose très prononcée, hypertrophie des nerfs périphériques). Mais ils s'éloignaient du type clinique par: d'une part, l'absence des douleurs fulgurantes, d'ataxie, des signes d'Argyll-Robertson et de Romberg vrais; d'autre part, la présence de tremblement intentionnel et de troubles de la parole se rapprochant de ceux de la sclérose en plaques.

L'étude anatomique d'un cas observé par Pierre Marie fut faite par Boveri qui, procédant à une étude comparative des obscrvations jusqu'alors publiées, a proposé, en 1910, la distinction de deux types cliniques de la névrite hypertrophique: le type Gombault-Déjerine et le type Pierre Marie. Il a donné le tableau comparatif et différentiel suivant:

\section{Type Gombrult-Dijerime}

Signe de Romberg.

Myosis.

Signe d'Argyll-Robertson.

Douleurs fulgurantes.

Ataxie des mouvements.

Contractions fibrillaires.

Nistagmens.

Absence de tremblement intentionnel.

Absence de la parole saccaldée.

Absence d'exophtalmic.

Atrophic musculaire généralisée.

\section{T'ylu" Pierre Marie}

Pas de signe de liomber vrai.

l'as de myosis.

l'as de signe d'Argyll-Robertson vrai, mais seulement paresse du róflexe pupillaire à la humière.

Pas de douleurs fulgurantes.

Pas d'ataxie des mouvements.

las de contractions fibrillaires.

Pas de nystagmus vrai.

'Tremblement intentionnel.

l'arole saccadée.

Exophtalmie.

Atrophie masculaire assez marquée à la jambe et extrêmement limitée à la main. 
Depuis lors, ont été fixées les caractéristiques essentielles des deux types classiques de la névrite hypertrophique progressive. Dans les deux modalités cliniques, l'incidence familiale et le début dans l'enfance représentaient des caractères fondamentaux pour la définition de la maladie, à côté de l'amyotrophie, de l'hypertrophie des nerfs et des troubles associés mentionnés.

Des observations postérieures ont cependant mis en évidence que la symptomatologie de la névrite hypertrophique n'est pas toujours aussi complète et définie que dans les cas de Déjerine et P. Marie; en effet, celle-ci peut, d'une part, simplement revêtir l'aspect seméiologique d'un processus névritique pur, et, d'autre part, le caractère familial et l'apparition précoce peuvent manquer. Ainsi, Long; déjà en 1907. puis en 1912, attirait l'attention sur une "forme atténuée" de la névrite hypertrophique en publiant deux cas qui avaient débuté à l'âge adulte, intéressant les membres supérieurs avec une disposition type Aran-Duchenne, sans troubles objectifs de la sensibilité, sans incoordination motrice ni anomalie pupillaire. Il n'y avait pas non plus d'augmentation appréciable du volume des nerfs, mais l'examen histologique a retrouvé une nette hypertrophie du tissu interstiriel et un épaississement annulaire de la gaine de Sclıwann, c'est-à-dire les lésions caractéristiques de la névrite interstitielle hypertrophique.

Aussi, en 1912, Hoffmann, à propos de cinq cas qu'il avait étudiés, soulignait que la symptomatologie de la névrite hypertrophique peut se réduirs à une modalité pluss purement périphérique, et le seul signe vraiment caractéristique de la maladie est l'hypertrophie des nerfs. Ces observations, avec celles du type Déjerine et du type Pierre Marie, formèrent le groupe des névrites hypertrophiques progressives.

Dans cette même série d'observations, Chiarini et Nazari ont publié, en 1913, l'étude clinique et anatomo-pathologique d'un cas de névrite hypertrophique qui présentait la symptomatologie de l'amyotrophie CharcotMarie. Il n'y avait pas d'antécédents familiaux et la maladia avait commencé à l'âge de 5 aus. Le diagnostic clinique de névrite hypertrophique ne pouvait pas être porté, disent les auteurs, parce que manquaient la cypho-scoliose et les symptômes tabétiques. L'épaississement, uniforme. était tellement peu prononcé qu'il aurait pu facilement passer inaperçu à l'autopsie si l'on n'avait pas trouvé quelques épaississements nodulaires dans les nerfs du membre supérieur.

En 1918, Dide et Courjon ont rapporté un cas de névrite hypertrophique de l'adulte sans antécédent familial, ayant débuté par une atrophie des membres supérieurs. En étudiant le même cas, Roussy et Cornil (1919) ont pratiqué une biopsie du cubital dont les résultats ont prouvé l'exactitude du diagnostic.

En 1926, Souques a publié un cas ayant débuté dans l'enfance et probablement familial, essentiellement caractérisé par des amyotrophies et hypertrophies des nerfs, avec une évolution progressive. L'absence de signes de la série tabétique, de la série sclérose en plaques, de la cyphoscoliose, le distinguait des types classiques de Combault-Déjerine et Marie Boveri. 
Ultérieurement, plusieurs auteurs rapportèrent des cas analogues aux différentes modalités signalées, contribuant, ainsi, à une meilleure connaissance des aspects cliniques de l'affection. À ce propos, Dechaume, Paillard et Ferragni, en 1934, ont classé les types cliniques suivant la symptomatologie (formes frustes, formes simples et formes compliquées), selon la date de début (formes de l'enfance et de l'adulte, de séméiologie simple ou compliquée) et selon l'évolution.

En ce qui concerne l'évolution de la névrite hypertrophique, une "forme récurrente" de la maladie a été décrite par Rossolimo, en 1912, basée sur un cas qui présentait "un cours quoique lent et progressif, mais compliqué de trois exacerbations des symptômes, toutes les fois reproduits d'une manière stéréotypée". Aussi Natras, en 1922, signalait l'observation d'une névrite hypertrophique récurrente, et Harris et Newcomb, en 1929, publiaient également une observation anatomoclinique très instructive. En 1935, Harris, à propos de cinq cas de polynévrites chroniques progressives, endotoxiques, accompagnées ou non d'hypertrophie des troncs nerveux, attirait de nouveau l'attention sur la possibilité d'évolution avec récurrences. Dans un cas de Diddle et Stephens (1936), une rémission du processus pendant un an a été observée.

$\mathrm{Du}$ point de vue anatomo-pathologique, les travaux de Marie et Bertrand de Bertrand et Souques, de De Bruyn et Stern ont bien établi les modalités fondamentales des lésions qui touchent la gaine de Schwann: la schwannite hypertrophique lamellaire (Souques et Bertrand, 1921), les "plasmatic swellings" (De Bruyn et Stern, 1929), la schwannite hypertrophique fibrillaire (Souques et Bertrand, 1934). Cornil, Chalnot, Railleanu ế Thomas (1930), ayant étudié un cas anatomo-clinique où il existait des phénomènes dégénératifs très importants de la gaine de Schwann contrastant avec une atteinte minime des cylindraxes et du tissu interstitiel, ont proposé la dénomination de "Schwannose hypertrophique progressive".

Enfin, le problème de la parenté entre la névrite hypertrophique avec certaines affections hérédo-dégénératives, surtout avec l'atrophie de Charcot-Marie, a suscité, dəpuis les premières publications de Déjerine, de nombreuses questions. Marinesco, Raymond, Beduschi ont défendu l'identité des deux processus; Austregésilo a signalé des cas avec symptomatologie de transition entre l'atrophie Charcot-Marie, la névrite hypertrophique et la maladie de Friedreich; plus récemment, André van Leeuwen, qui a très bien étudié les troubles pupillaires dans les affections hérédo-dégénératives du système nerveux (1942-1946), et également François et Descamps (1949), concluent sur l'unicité des deux affections, qui devraient être réunies sous la dénomination d'amyotrophie neurale hérédo-dégénérative, "le même gène donnant lieu à des manifestations phénotypiques légèrement différents". À ce propos, L. van Bogaert écrit: "La parenté étroite, non seulement clinique, mais anatomique, de la forme classique de Charcot-Marie-Tooth et de la polynévrite hypertrophique de Déjerine-Sottas ne fait plus de doute. Il s'agit, dans les deux cas, d'un processus atteignant les nerfs sensitivo- 
moteurs, avec cette différence que, dans la seconde, l'endonèvre et l'appareil schwannique montrent une réaction d'une densité telle que certains auteurs vont jusqu'à la considérer comme néoplasique".

\section{SYMPTOMATOLOGIE}

Le tableau clinique de la névrite hypertrophique comporte: 1) l'hy. pertrophie des nerfs, les amyotrophies, l'aréflexie tendineuse, qui constituent les manifestations cliniques les plus caracteristiques de la maladie; 2) des troubles associées, de plusieurs types: syndrome sensitif, syndrome oculaire, syndrome cérébelleux, syndrome végétatif, troubles trophiques et dysplasiques.

L'hypertrophie des nerfs, qui constitue la caractéristique principale de la maladie, est retrouvée fréquemment dans la partie tronculaire du nerf (cubital, médian, sciatique poplité exterme) et également au niveau des rameaux sous-cutanés (du radial, du plexus cervical superficiel, etc.). L'hypertrophie du crural a été observée par Cornil et Chalnot, celle du spinal par Radermecker, des intercostaux, et du sus-orbitaire par Tarassiewitch. L'importance de l'hypertrophie des rameaux nerveux sous-cutanés a été soulignée par P. Marie et bien décrite par Boveri. Les nerfs hypertrophiés ont généralement une consistance augmentée; ils sont durs et indolores. L'épaississement est fréquemment uniforme et régulier; l'aspect fusiforme et des nodosités sont néanmoins signalés dans quelques observations (Rossolimo, Cornil et collaborateurs, Chiarini et Nazari). L'hypertrophie peut atteindre un volume considérable dans quelques cas ("neuromegalia hypertrophica progressiva", de Tarassiewitch), et les branches sous-cutanées peuvent être directement perceptibles à la vue, comme une espèce d'arborisa. tion nerveuse (Boveri); d'autres fois, elle est tellement modérée qu'elle peut passer inaperçue (cas de Long, de Chiarini et Nazari).

Syndrome amyotrophique - Comme dans l'amyotrophie type Charcot-Marie-Tooth, la névrite hypertrophique progressive est plus amyotrophique que paralytique; Souques, particulièrement, a insisté sur ce point. Dans les cas anciens, néanmoins, la déficience motrice peut-être totale. surtout au niveau des membres inférieurs. Les atrophies musculaires, de topographie généralement distale, réalisent aux membres supérieurs l'aspect Aran-Duchenne et aux membres inférieurs l'aspect Charcot-Marie. Garcin a insisté sur le fait que l'amyotrophie ne frappe d'abord et pendant assez longtemps qu'un seul membre avant de s'étendre aux autres segments des années après (névrites multiples successives). Dans quelques cas, l'atrophie s'étend aux membres proximaux (Long, Achard et Thiers). Des fibrillations sont mentionnées dans quelques observations. La réaction de dégénérescence accompagne les amyotrophies, et Souques et Duhem en ont faite une étude comparative dans l'atrophie Charcot-Marie et dans la névrite hypertrophique. 
L'aréflexie tendineuse, un des signes les plus constants de l'affection, est précoce et d'ordinaire généralisée.

Syndrome sensitif - Des paresthésies et des douleurs, du type fulgurant, lancinant ou névralgique, sont fréquentes, et une forme douloureuse de la névrite hypertrophique a même été signalée par Dawidenkow, en 1939. Le syndrome douloureux était important dans les cas de Cornil el collaborateurs, De Bruyn et Stern, Wolff, Rubinowitz et Burchell, Sloane et Tarassiewitch. Dans quelques-uns de ces cas, les douleurs radiculaires simulaient le tabès et, dans le cas de Delhaye et van Bogaert, les douleurs gastriques rappelaient l'ulcère de l'estomac.

Les troubles de la sensibilité objective superficielle sont en général modérés et peuvent même être ignorés du malade. Ils sont d'habitude la découverte d'un examen neurologique systématique. Dans les observations princeps de Déjerine cependant, "il existait des troubles extrêmement marqués de la sensibilité, avec retard dans la transmission des impressions, troubles plus accusés aux membres inférieurs qu'aux membres supérieurs et diminuant d'intensité de la périphérie au centre". Les troubles sensitifs superficiels revêtent d'habitude une topographie tronculaire ou une topographie segmentaire, due à l'atteinte plurinévritique.

La sensibilité profonde est, d'autre part, fréquemment lésée, surtout la sensibilité au diapason et la notion de position des membres. Lié à cette atteinte de la sensibilité profonde, on constate souvent un syndrome ataxique important qui, avec l'aréflexie tendineuse, les douleurs fulgurantes, les troubles oculaires, réalise un véritable syndrome tabétique (cas de Gombault et Mallet, de Déjerine-Sottas, de A. van Leeuwen et Moreau). L'ataxie est surtout statique, ou à la fois statique et dynamique.

Syndrome oculaire - Il consiste principalement en troubles pupillaires (altérations de la forme et des dimensions des pupilles, troubles du réflexe photomoteur, signe d'Argyll-Robertson, syndrome pupillotonique, etc.) qui ont été signalés dans $40 \%$ des cas publiés de névrite hypertrophique, selon François et Descamps.

Le signe d'Argyll-Robertson a été retrouvé par plusieurs auteurs (Déjerine, Wolf, Rubinowitz et Burchell, Delhaye et van Bogaert, etc.). De Bruyn et Stern ont minimisé l'intérêt de ce signe dans la névrite hypertrophique en la rattachant à la syphilis. Cependant, les constatations de plusieurs auteurs ont montré l'importance et la fréquence de ce signe dans la névrite hypertrophique en dehors de toute syphilis associée. Ainsi, dans le cas anatomo-clinique de Sloane, où il existait un signe d'Argyll-Robertson, on a retrouvé une dégénérescence grave des noyaux des colliculi antérieurs (tectum mésencéphalique), et aucune lésion anatomique de syphilis n'a été révélée.

A. van Leeuwen a insisté, en particulier, sur la diversité des troubles pupillaires rencontrés dans l'amyotrophie neurale et sur l'analogie que pré- 
sentent les différentes formes cliniques avec celles produites par l'infection syphilitique. Elle a signalé que les trois types de troubles pupillaires que Behr décrivit dans le tabès dorsal peuvent être observés dans l'amyotrophie neurale: "L'atteinte isolée, simples, du réflexe photomoteur; une abolition du réflexe photomoteur faisant partie d'un syndrome véritable d'Argyll-Robertson; enfin, une atteinte des réflexes photomoteurs accompagnée d'une tonicité marquée des syncinésies pupillaires. Ce dernier syndrome prend une signification toute particulière du fait qu'il doit être considéré comme un chaînon reliant directement la pupillotonie authentique (décrite dans une observation isolée de névrite hypertrophique par Storring) aux manifestations pupillaires de la névrite hypertrophique". A. van Leeuwen a encore souligné que les troubles pupillaires se manifestent de préférence chez des sujets présentant l'hypertrophie nerveuse et des troubles de la série tabétique (crises gastriques, incoordination, douleurs lancinantes).

François et Descamps, qui ont réalisé récemment une étude systématique des altérations pupillaires dans la névrite hypertrophique, sont arrivés aux conclusions suivantes:

“1) L'atteinte principale porte sur le réflexe photomoteur. Il s'agit d'abord d'un simple affaiblissement ou d'une certaine lenteur avec diminution d'amplitude. Ce trouble, le plus précoce, toujours isolé au début, et pouvant le rester, s'aggrave et évolue plus ou moins rapidement vers l'aréflexie photomotrice totale. 2) Celle-ci peut coexister avec toute la gamme des dimensions pupillaires; toutefois, elle s'accompagne de préférence d'un rétrécissement de la pupille; celui-ci peutètre progressif et l'on peut à la longue observer un myosis franc avec dimensions pupillaires invariables. 3) Le réflexe à l'accommodation-convergence est fréquemment conservé, même après trente ou quarante ans d'évolution. Il est normal, et parfois même exagéré. Parfois, cependant, il s'entreprend également. L'atteinte se fait alors soit dans le sens d'une simple paresse ou d'une diminution d'amplitude, soit plus rarement dans le sens d'une pupillotonie. Il est exceptionnel de voir l'atteinte simultanée des réflexes à la lumière et è la convergence évoluer vers une fixité pupillaire plus ou moins totale. Storring a décrit un cas de pupillotonie vraie et $\mathrm{A}$. van Leeuwen a observé un cas de pupillotonie atypique. 4) Les pupilles peuvent avoir une forme régulière; mais très souvent elles se déforment assez fortement et plus ou moins rapidement pour dèvenir ovalaires ou triangulaires. L'anisocorie est la règle et se manifeste d'une façon précoce. 6) Le réflexe orbiculaire est conservé, mais le réflexe pathique ou psycho-sensoriel est généralement aboli. 7) Les mydriatiques ne produisent qu'une dilatation très modérée chez les sujets dont la pupille est manifestement atteinte".

En dehors des anomalies pupillaires, d'autres troubles oculaires ont été également signalés dans la névrite hypertrophique: un oedème pupillaire, dans le cas de Diddle et Stephens; une décoloration du segment temporal de la papille, dans le cas de Souques; un rétrécissement du champ visuel, dans le cas de Wolf, Rubinowitz et Burchell.

Syndrome cérébelleux - Les éléments d'un syndrome cérébelleux (tremblement intentionnel et parole scandée) sont plus rarement constatés, mais ils ont été signalés dans quelques observations. Ils étaient particulièrement importants dans les cas étudiés par P. Marie et Boveri et caracté- 
risent la forme clinique décrite par ces auteurs. Selon A. Thomas et Chausseblanche, il s'agirait, dans ces cas, d'une association de deux processus: névrite hypertrophique et processus dégénératif responsable de la séméiologie cérébelleuse.

Syndrome végétatif -.. Des troubles végétatifs (sudation spontanée exagérée, troubles vasomoteurs, exophtalmie) ont été signalés dans quelques observations: P. Marie, Boveri, van Bogaert, etc.

Syndrome trophique - Rarement observés, les troubles trophiques sont mentionnés dans les observations de Cornil, de Hesse et de Lesniowski. Celui-ci a rapporté deux cas de névrite hypertrophique chez le père et le fils, avec existence de troubles trophiques des membres inférieurs chez le père allant jusqu'à la perte d'un orteil.

Un cas d'amyotrophie des membres à prédominance distale, accompagnée d'hypertrophie des nerfs cubitaux avec rétractions fibrotendineuses des doigts et des mulilations des orteils, a été publiée par Thiebault et ses collaborateurs. La biopsie du cubital n'ayant pas montré les "bulbes d'oignon" caractéristiques de la névrite hypertrophique, les auteurs ont discuté l'origine lépreuse possible de cette névrite.

Troubles dysplasiques - Des stigmates dysraphiques ont été signalés par A. van Leeuwen, des déformations du crâne par Villaret, Haguenau et Klotz. La cypho-scoliose est souvent retrouvée, ainsi que des déformations des pieds (pieds creux et pieds bots congénitaux).

Altérations moins fréquentes - Il existait un signe de Babinski, dans les cas de Villaret, Haguenau, Klotz, et de Achard-Thiers; une parésie passagère du droit externe (à l'occasion des exacerbations de la maladie) dans le cas de Rossolimo; des troubles sphinclériens importants dans le cas de van Rellin; des troubles psychiques dans les cas de Déjerine-Sottas, De Bruyn et Stern, François et Descamps, etc.

Liquide céphalo-rachidien - Il est habituellement normal, mais une hyperalbuminose importante existait dans le cas de Diddle et Stephens.

\section{FORMES CLINIQUES}

Les observations publiées de névrite hypertrophique montrent la diversité d'aspects séméiologiques et des tableaux cliniques que peut revêtir l'affection: on distingue, à côté des formés complètes, douées d'une symptomatologie mixte périphérique et centrale, des formes simples, dont la symptomatolog:e correspond exclusivament à un processus névritique pur.

Les formes simples, ou névritiques pures, se caractérisent essentiellement par le syndrome amyotrophique-neurohypertrophique, avec aréflexie tendineuse. Des troubles généralement discrets de la sensibilité objective 
sont souvent observés. Les cas sporadiques de névrite hypertrophique prennent fréquemment cet aspect clinique (observations de Hoffmann, Long, Chiarini-Nazari, Dide-Courjon et Roussy-Levy, Souques, etc.). Les troubles débutent le plus habituellement à l'âge adulte; néanmoins, dans l'observation de Chiarini et Nazari, la maladie avait commencé à 5 ans.

Dans les formes complètes, ou combinées, le complexus amyotrophieneurohypertrophie-aréflexie constitue également la base de la symptomatologie; mais il existe, en plus, des troubles de la série tabétique (névrite hypertrophique type Déjerine-Sottas) ou de la série cérébelleuse (type $P$. Marie-Boveri). Les troubles débutent en général dans l'enfance et le caractère familial est souvent évident.

\section{DIAGNOSTIC}

En plus de l'hypertrophie des nerfs, qui constitue le signe majeir de maladie, l'amyotrophie et l'aréflexie sont également des éléments de primordiale importance pour le diagnostic. L'installation à des périodes successives des amyotrophies et leur évolution très lentement progressive sont des caractères qui doivent retenir l'attention (névrites multiples et successives).

Au point de vue du diagnostic différentiel, il se pose essentiellement avec l'amyotrophie type Charcot-Marie. Les ressemblances des deux tableux cliniques sont frappants, et seule l'hypertrophie des nerfs permet souvent la distinction. D'ailleurs, certains auteurs (Marinesco, Raymond, Beduschi, van Leeuwen, etc.) défendent l'unicité des deux tableaux: ce seraient deux formes cliniques d'une même maladie. A. van Leeuwen se base sur des documenís histologiques et sur des arguments d'ordre génétique: coexistence dans une même famille de l'atrophie Charcot-Marie et de la névrite hypertrophique. François et Descamps soutiennent également que, dans les cas qu'ils ont étudiés, il est impossible d'isoler un tableau séméiologique précis; ils ont trouvé, dans l'un et l'autre tableau, une "interpénétration des symptômes". Van Bogaert signale, à ce propos, que: "La parenté étroite, non seulement clinique, mais anatomique, de la forme classique de Charcot-Mar:e-Tooth et de la polynévrite hypertrophique de Déjerine et Sottas ne lait plus de doute. Il s'agit, dans les deux cas, d'un processus atteignant les nerfs sensitivo-moteurs, avec cette différence que, dans la seconde, l'endonèvre et l'appareil schwannique montrent une réaction d'une densité telle que certains auteurs vont jusqu'à la considérer comme néoplasique".

Le diagnostic avec la maladie de Friedreich repose essentiellement, d'une part, sur la présence du syndrome amyotrophique-neurohypertrophique et, d'autre part, sur l'absence de signes pyramidaux. Un des cas de névrite hypertrophique publié par Déjerine en 1893 avait été catalogué, trois ans auparavant, comme forme spéciale de maladie de Friedreich. 
La lèpre nerveuse peut soulever des difficultés diagnostiques dans les pays où le Mal de Hansen est endémique: en effet, il s'agit dans les deux maladies d'un tableau de névrite multiple hypertrophique et progressive. Dans la névrite lépreuse, le processus est cependant en général moins diffus, avec atteinte élective du cubital, médian, sciatique poplité externe et facial. D'autre part, l'atteinte de la sensibilité superficielle est beaucoup plus importante que dans la névrite hypertrophique; une anesthésie absolue à tous les modes est fréquente. Les troubles trophiques sont également plus fréquents et sévères. Par contre, l'aréflexie tendineuse précoce et généralisée n'est pas d'observation habituelle dans la lèpre. Dans les cas douteux, la biopsie transchera le diagnostic, puisque les lésions histologiques sont totalement différentes: schwannite dans la névrite hypertrophique, lésions inflammatoires du type lépromateux, tuberculoïde ou indifférencié dans la lèpre. nerveuse.

Le diagnostic avec d'autres polynévrites se base essentiellement sur: l'évolution (plus rapide) et le mode d'installation du processus, qui est massive, globale, d'emblée et symétrique dans les vraies polynévrites; d'autre part, sur l'absence de neurohypertrophie (il importe de signaler, cependant, les observations de W. Harris sur les polynévrites chroniques progressives endotoxiques, qui peuvent s'accompagner d'un épaississement des nerfs); enfin, sur les symptômes associées que nous avons déjà mentionnés et qui ne se rencontrent pas dans les polynévrites habituelles.

La forme classique de Déjerine et Sottas soulève un diagnostic avec le tabès amyotrophique. L'hypertrophie nerveuse, le caractère familial et, d'autre part, l'examen du sang et du liquide céphalo-rachidien, feront la distinction.

Le diagnostic se pose enfin avec d'autres affections où une hypertrophie des nerfs peut exister: névrite sclérogommeuse syphilitique (Guillain et Perisson), hypertrophie localisée des troncs nerveux post-traumatique (Alajouanine, d'Allaines et Saucier), neurofibromatose (dans un cas de "neurofibromatose localisée, disposée en ceinture thoracique", étudié par Souques, Alajouanine et Lermoyez, ces auteurs ont trouvé une hypertrophie des nerfs intercostaux, avec un aspect moniliforme). D'ailleurs, les rapports de la névrite hypertrophique avec la neurofibromatose ont attiré l'attention de nombreux auteurs (Hoffmann, Bielchowski, Dawidenkow, Slauck); pour Dawidenkow, la névrite hypertrophique serait même une forme de maladie de Recklinghausen.

RESUMO

Três observações de neurite hipertrófica progressiva são apresentadas. A primeira refere-se a um caso esporádico da moléstia, o início tendo-se processado aos 28 anos, não existindo quaisquer antecedentes familiais. Os distúrbios observados (amiotrofias, neuro-hipertrofia, arreflexia profun- 
da e discretas alterações das sensibilidades superficiais) e, por outro lado, a ausência de manifestações do tipo tábido ou cerebelar, permitem classificar o caso no grupo das neurites hipertróficas simples. E' de notar-se o caráter múltiplo e sucessivo com que se instalaram as atrofias musculares, tendo sido afetados, inicialmente, os membros superiores.

$\mathrm{Na}$ observação 2 trata-se igualmente de um caso esporádico da moléstia, com sintomatologia essencialmente periférica (forma simples da N.H.), e na qual se destacam a idade de início (18 anos), a ausência de caráter familial, o aspecto múltiplo e sucessivo com que ocorreram as atrofias musculares (apresentando-se, inicialmente, nos membros inferiores), a ausência de sinais da série tábida ou cerebelar.

A observação 3 refere-se a um paciente, em cuja família são encontradas referências numerosas a distúrbios neurológicos. $O$ caso estudado (IV-3) e uma das irmãs do paciente (IV-6) - e provàvelmente os outros membros assinalados - apresentam, essencialmente, um quadro amiotrófico associado a sinais da série tábida. A hipertrofia dos nervos periféricos foi verificada nos casos IV-3 e IV-6, estabelecendo-se assim o diagnóstico de neurite hipertrófica progressiva tipo Déjerine-Sottas. A pesquisa do espessamento de nervos não pôde, infelizmente, ser investigada nos outros membros da família. No caso 3, é de notar-se ainda a idade em que tiveram início as manifestações mórbidas (aos 40 anos, aproximadamente), diferindo, nisto, dos casos clássicos de Déjerine, de início na infância ou adolescência.

A biópsia de um ramo superficial do nervo radial foi praticada nos casos 1 e 2, tendo o exame histopatológico evidenciado as lesões características da schwannite hipertrófica, acompanhadas de degenerações mielínicas e axônicas mais ou menos intensas, lentamente evolutivas.

$O$ autor expõe, a seguir, os principais dados relativos ao histórico, sintomatologia, formas clínicas e diagnóstico. Analisa as manifestações básicas do quadro clínico (amiotrofias, estado hipertrófico dos nervos, arreflexia profunda) e as manifestações associadas (síndromes sensitiva, cerebelar, vegetativa, trófica e displástica). Quanto às formas clínicas, distingue: 1) as formas simples, ou neuríticas puras, essencialmente caracterizadas pela síndrome amiotrófica-neurohipertrófica, arreflexia profunda e discretas desordens das sensibilidades superficiais; 2) as formas completas ou combinadas, nas quais, à associação amiotrofia-neurohipertrofiaarreflexia, superajuntam-se distúrbios da série tábida (tipo Déjerine-Sottas) ou cerebelar (tipo Marie-Boveri). Enquanto que os casos esporádicos de N.H. assumem geralmente a primeira forma, os casos com incidência familial, de início na infância, revestem o segundo aspecto na maior parte das vêzes.

Ao que parece, a afecção passa muitas vêzes despercebida, confundida com síndromes amiotróficas e atáxicas de outra natureza, em virtude de não se proceder, no exame neurológico habitual, à pesquisa sistemática da hipertrofia dos nervos periféricos. 


\section{SUMMARY}

Three cases of Progressive Hypertrophic Neuritis are reported. The first refers to a sporadic case of the disease which began when the patient was 28 years old. The disturbances observed (muscular atrophies, thickening of peripheral nerves, loss of tendon reflexes and slight disorders of cutaneous sensibilities) and the absence of manifestations of tabetic or cerebellar type classify the case into the group of the simple hypertrophic neuritis. The atrophies commenced in the left hand and developed in a multiple and successive character.

The second case also deals with a sporadic form of the disease with essentially peripherical symptomatology (simple form of the N.H.). The illness started at 18 years of age; no familial incidence; the muscular atrophies occurred in a multiple and successive manner beginning in the left leg; the signs of the tabetic or cerebellar group were absent.

The third case refers to a patient in whose family numerous references to neurological disturbances are encountered. The case studied (IV-3) and one of the sisters of the patient (IV-6) and probably the other indicated members of the family present essentially an amyotrophic picture associated to the signs of the tabetic group. The thickening of the peripheral nerves was verified in cases IV-3 and IV-6, thus establishing the diagnosis of hypertrophic neuritis, Déjerine-Sottas' form. The research of thickening of nerves unfortunately could not be investigated in the other members of the family. In case 3 the disturbances commenced at approximately 40 years of age, differing from the classic cases of Déjerine beginning in childhood or adolescence.

A biopsy of superficial branch of the radial nerve was performed in cases 1 and 2 and the histopathological examination revealed the characteristic lesions of "schwannite hypertrophique" ("onion bulbs").

The principal elements concerning the historical of the disease, symplomatology, clinical forms and diagnosis are described later. The cardinal feature of the disorder (amyotrophies, thickening of peripheral nerves, loss of tendon reflexes) and the associated symptoms and signs (sensory, ocular, cerebellar, trophic changes, etc.) are analysed. Regarding the clinical forms, the author distinguishes: 1) the simple forms, or purely neuritic, characterized by the progressive amyotrophy of distal limb segments, thickening of peripheral nerves, loss of tendon reflexes and impairment of cutaneous sensibility; and 2) the complete or combined form, in which the disorders of tabetic or cerebellar group add to those previously referred to. While the sporadic cases of H.N. generally assume the first form, the familial cases, beginning in childhood, usually reveal the second form.

It seems that the diseases is sometimes not recognized, confused with amyotrophic and ataxic syndromes of another nature, because the research of the enlargement of peripheral nerves is not always accomplished in the habitual neurological examination. 


\section{BIBLIOGRAPHIE}

1. Alajouanine, D'Allaines et Saucier - Fracture spontanće de l'humćrus, premier signe d'une syringomyélie. Hýpertrophie localisée des trones nerveux posttraumatiques. Rev. Neurol., 1:76-84 (Janvier) 1927.

2. Austregésilo, $\Lambda$. - Parenté entre l'atrophie musculaire Charcot Marie, Névrite de Déjerine-Sottas et Maladie de Friedreich. Rer. Sucl-am. Méd. Chir., 1:247-261 (Mars) 1930 .

3. van Bogaert, L. - Ia polynévrite hypertrophique progressive familiale de Déjerine-Sottas. 'I'raité de Médicine, Masson et Cie., Paris, tome 16, pag 174-180, 1949.

4. Borges-Fortes, A. et Magallães, E. - Sôbre um caso de nevrite hipertrófica com exame histopatológico. Arq. Bras. Neuriat. e Psiquiat, 15:29-37, 1933

5. Boveri, P. - De lin névrite hypertrophique familiale (tỵpe l'ierre Marie). Semaine Véd., no 13, pag. 145-150 (30 Mars) 1910.

6. Busscher, J. De et Bogaert, L. van - Sur la polynécrite familiale hypertrophique progressive (type fruste de l'adulte). J. Bielge de Neurol. at P'sychiat., 35:152-169, 1935.

7. Chiarini, P. et Nazari, A. - Studio clinico e anatono-patologico di un caso di nevrite interstiziale ipertrofica con atrofia nuscolare tipo Charcot Marie. Riv, Ospedaliera, 3:185-202 (15 Marzo) 1913.

8. Cornil, Chalnot, Kaileanu et Thomas - Névrite hipertrophique progressive non faniliale (étude anatomo-clinique). Rer. Neurol., 1:1187-1192 (Juin) 1930.

9. Dechaume, J., Paliard, F. et Ferragne, G. - I a névrite interstialle hypertrophique et progressive. Schwannite hyperplasique neuro-hypertrophiante. J. Méd, de Lỵon, 15:829-848 (20 Décembre) 1934.

10. Delhaye, A. et Bogacrt, 1 . van - Un cas de névrite hypertrophique familiale. J. Belge de Neurol. et de Psỵchiat., 34:341-344, 1934.

11. Déjerine, $\mathbf{J}$. et sottas - Sur la névrite interstitielle hypertrophique et progressive de l'enfance. Mém. Soc. de I3iol., 5:63-96, 1893.

12. Déjerine, J. - Contribution à l'étude de la névrite interstitielle hỵvertrophique et progressive de l'enfance. Scmaine Méd., pàg. 491, 1896.

13. Dide, Y. - I a névrite hỵpertrophique de l'adulte. Rev. Neurol, 35:934$935,19 i \jmath$.

14. Dide et Courjon - - I) Un cas de névrite hypertrophique de ladulte. Nouvelle Iconographie de la Salpétrière, 28:377, 1918. b) I a névríte hypertrophique de l'adulte. Rev. Neurol., 35:825, 1919.

15. Diddle, A. W. et Stephens, R. I. -- Hypertrophic interstial neuritis with papilledema. Arch. Neurol, a. Psychiat., 40:151-157 (Juillet) 1938.

16. Ferragne, G. - La névrite interstitielle hypertrophique et progressive. Schwannite hyperplasique neuro-hypertrophiante. Etude anatomo-clinique. Thèse de Lyon, 1932. Edit. Bosc Frères.

17. François, J. et Descamps, L. - Etude neuro-ophtalmologique de deux souches d'amyotrophie neurale hérédodégénerative, l'une du type Charcot-Marie'Tooth, l'autre du tỵpe névrite hỵpertrophique de Déjerine-Sottas. Acta Neurol. et Psychiat. Belgica, 49:648-676 (Septembre) 1949.

18. Gallotti, $O$. - Forme spéciale de la maladie de Friedreich ou nouvelle maladie familiale. Rev. Neurol., 2:758-764 (Novembre) 1928.

19. Garcin, R., Bertrand, I., Freitas Julião, O. et Buge - Sur deux cas sporadiques de névrite hỵpertrophique progeressive de Déjerine-Sottas avec contrôle biopsique. Rev. Neurol., 82:204-210 (Mars) 1950.

20. Gombault et Mallet - Un cas de tabès ayant débuté dauns l'enfance; au topsie. Arch. de Méd. Exper. et d'Anat. Pathol., 1:385, 1889. 
21. Guillain, G. et Perisson - Névrite chronique hypertrophique scléro-gommeuse du nerf cubital chez un syphilitique tabétique. Rev. Neurol., 1:27, 1931.

22. Harris, W. - Chronic progressive (endotoxic) polyneuritis. Brain, 58:368$375,1935$.

23. Hoffmann, J. - Über progressive hypertrophische Neuritis. Deutsche Ztschr. f. Nervenh., 44:65-94, 1912.

24. a) van Leeuwen, A. - De la valeur des troubles pupillaires, en dehors de la syphilis, comme signe précoce ou forme fruste d'une affection hérédodégénerative. Monatschr. f. Psychiat. u. Neurol., 105:193-216, 1942; 105:275299, 1942; 108:1-89, 1943. b) van Leeuwen, A. et Moreau, M. -- Idem. Monatschr. f. Psychiat. u. Neurol., 112:121-168, 1946.

25. Lesniowski, St. - Névrite interstitielle progressive. Res. in Rev. Neurol., 1:367, 1931.

26. Lesniowski, S. - Névrite hypertrophique progressive chez le père et le fils (type Hoffmann). Polska Gazeta Lekarska, vol. 11, $\mathrm{n}^{\circ} 39$ (Septembre) 1932. Res. in Presse Méd.

27. Long, E. - Atrophie musculaire progressive type Aran-Duchenne de nature névritique. Nouvelle Iconographie de la Salpétrière, 25:281-308, 1912.

28. Marie, P. - Forme spéciale de névrite interstitielle hypertrophique progressive de l'enfance. Rev. Neurol,, 14:557, 1906.

29. Massion-Verniory, L. et Radermecker, J. -- Névrite hypertrophique interstitielle. Monatschr. f. Psychiat. u. Neurol., 114:246-257, 1947.

30. Rossolimo, G. -- Sur une forme récurrente de la polynévrite interstitielle hypertrophique progressive de l'enfance (Déjerine) avec participation du nerf oculo-moteur externe. Rev. Neurol., 7:558-564 (Aoit) 1899.

31. Roussy, G. et Cornil, L. - Un cas de névrite hypertrophique progressive non familiale de l'adulte. Rev. Neurol., 35:590-592 (Juillet) 1919.

32. Saucier, J. - La Névrite Hypertrophique. Thèse de Paris, 1927. Ed. Arnette.

33. Schaller, W. F. et Newmann, H. W. - Névrite interstitielle hypertrophique. Rev. Neurol., 1:529-539, 1935.

34. Sloane, P. - Progressive interstitial hypertrophic neuritis. J. Nerv. a. Ment. Dis., 90:429-438, 1939

35. Souques, A. - Forme atypique de névrite hypertrophique progressive. Ann. de Méd., 19:484-496 (Mai) 1926.

36. Souques, A. et Bertrand, I. - Un cas anatomo-clinique atypique de névrite hypertrophique progressive de l'enfance. Rev. Neurol., 2:513-530, 1934.

37. Souques, Alajouanine et Lermoyez - Neurofibromatose localisée, disposée en ceinture thoracique. Bull. et Mém. Soc. Méd. Hôp. Paris, 45:1729-1733, 1921.

38. 'Tarassiewitch, I. J. et Michejew, W. - Névrite hypertrophique et progressive (Neuromegalia peripherica progressiva). Rev. Neurol., 64:18-40 (Juillet) 1935 .

39. 'Ihiebaut, F., Wolinetz, E., Daum, S. et Fenelon, F. - Amyotrophie à prédoninance distale avec mutilation des orteils, rétraction fibro-tendincuse des doigts et hypertrophie des nerfs cubitaux. Discussion de l'origine lépreuse de cette névrite hypertrophique. Rev. Neurol., 79:438-442 (Juin) 1947.

40. 'Ihomas, A. et Chausseblanche, L. - Un cas de névrite hypertrophique et progressive de l'enfance. Encéphale, 28:504-530, 1933.

41. Villaret, M., Haguenau, J. et Klotz, P. H. - Névrite hypertrophique familiale. Rev. Neurol., 63:211-218, 1935.

42. Wolf, A., Rubinowitz, O. H. et Burchell, S. C. - Interstitial hypertrophic neuritis of Déjerine and Sottas. A report of three cases. Bull. Neurol. Inst. New York, 2:373-428 (Novembre) 1932. 